\title{
Criminologie
}

\section{Faut-il transformer ou abolir la probation?}

\section{André Normandeau}

Volume 12, numéro 2, 1979

Probation : aide ou contrainte?

URI : https://id.erudit.org/iderudit/017110ar

DOI : https://doi.org/10.7202/017110ar

Aller au sommaire du numéro

Éditeur(s)

Les Presses de l'Université de Montréal

ISSN

0316-0041 (imprimé)

1492-1367 (numérique)

Découvrir la revue

Citer ce compte rendu

Normandeau, A. (1979). Compte rendu de [Faut-il transformer ou abolir la probation ?] Criminologie, 12(2), 89-100. https://doi.org/10.7202/017110ar d'utilisation que vous pouvez consulter en ligne.

https://apropos.erudit.org/fr/usagers/politique-dutilisation/ 


\section{Introduction}

Il est d'usage pour un universitaire qui écrit un article sur une pratique criminologique, que ce soit la prison, les libérations conditionnelles, la police, les tribunaux, la délinquance des mineurs... ou la probation, d'être provoquant afin de susciter le débat. Le lecteur-praticien peut toujours alors mettre de côté de telles remarques provocatrices en se disant que l'universitaire est un théoricien, qu'à ce titre il est trop éloigné de la vraie vie et qu'en conséquence ce qu'il dít n'est pas pertinent pour le praticien.

Je suis prêt à reconnaître que ce jugement est de temps en temps correct car, effectivement, trop d'universitaires parlent * à travers leur chapeau * de choses qu'ils ne connaissent pas convenablement. Comme je n'ai pas l'intention de percer mon propre chapeau, je ne voudrais donc pas utiliser cette note de lecture pour me prononcer sur certains aspects de la pratique en probation pour adultes au Québec ou ailleurs.

Toutefois, je pense qu'il serait intéressant pour le praticien de replacer l'interrogation sur le rôle et la fonction de l'agent de probation au Québec sur la toile de fond plus vaste : a) de la remise en question du modèle médical au sein du système correctionnel adulte; b) des idées émises sur le rôle social et communautaire beaucoup plus étendu de la probation pour adultes.

Dans cette perspective, la littérature nord-américaine sur le sujet est très abondante et elle a l'avantage de s'inscrire dans une tradition criminologique très récente qui a fait appel souvent aux travaux et à l'expérience concrète des praticiens. Notre 4 note de lecture , voudrait de façon particulière attirer l'attention du lecteur québécois sur l'excellente revue américaine Corrections Magazine qui, dans le secteur correctionnel adulte, est à la criminologie ce que les revues Psychologie et Saciety (sociologie) sont aux disciplines psycho-sociologiques, c'est-à-dire une

* André Normandeau est professeur et directeur de l'Ecole de criminologie de l'Université de Montréal. 
revue de type populaire et vulgarisatrice pour l' \&onnête citoyen s et praticien intéressé à connaître rapidement et de façon vivante l'essentiel des grands débats et des expériences concrètes pertinents à sa vie professionnelle. En 1979, un criminologue ne peut pas se passer de Corrections Magazine et il ne s'agit pas là d'une forme de publicité mais plus simplement d'une conviction personnelle.

La revue Corrections Magazine est publiée depuis 1974. Elle traite de toutes les questions relatives au secteur correctionnel adulte, et même, à l'occasion, du secteur correctionnel pour les jeunes délinquants. Le monde des prisons a été jusqu'ici favorisé mais certaines idées novatrices au sujet de la probation ne peuvent être comprises qu'à la lumière de la critique radicale des dernières années du phénomène carcéral.

Nous voudrions donc, dans un premier temps, résumer le débat de fond à ce sujet, tel que présenté dans cette revue, et illustrer par la suite son impact sur le monde particulier de la probation.

\section{Prisons : le modèle médical versus le modèle de justice}

Nous ne voulons pas approfondir ce débat des modèles puisqu'il est maintenant relativement bien connu et que l'article d'Yvon Dandurand et de Jacques Laplante au sommaire du present numéro de la revue Criminologie y fait également allusion. Rappelons-en seulement, toutefois, les lignes maîtresses.

Un revirement d'opinion exceptionnel a eu lieu au cours des dernières années aux États-Unis, au Canada et au Québec, au sujet du traitement des criminels. Pendant près d'un siècle, la pénologie nord-américaine avait inscrit et accepté sans réserve l'idéal de la réhabilitation et du traitement; cet idéal qui lie le temps passé en prison par un criminel au pronostic et au progrès de la « guérison 》 de ses tendances criminelles. Jusqu’à la fin des années 60 , cette position representait le point de vue éclairé et les réformateurs réussirent souvent à introduire cette perspective au cœur de la politique criminelle. Puis soudainement, ce courant d'opinion se transforma en contre-courant. Une vague de scepticisme, et même l'hostilité ouverte, émergea, orientée contre la théorie et la pratique carcérale assises sur le socle de l'idéal de la réhabilitation et du traitement ; également, une 
vague de préoccupations an sujet de l'injustice qui caractérise souvent les relations entre fonctionnaires et détenus en milieu carcéral. Cette attaque contre la doctrine officielle de la criminologie du $x^{e}$ siècle s'est ralliée rapidement des supporteurs d'orientations politiques et sociales fort diverses; il n'est que de lire la littérature scientifique et populaire récente sur le monde des prisons, de la réhabilitation et du traitement, littérature fort abondante qu'il est impossible ici de résumer, pour se rendre compte à quel point l'idéal de la réhabilitation bat en retraite. De plus, plusieurs politiciens et plusieurs projets officiels de législations, en matière criminelle et pénale, s'inspirent dorénavant d'une telle approche résolument *agnostique * à l'égard du traitement des criminels.

Un des livres populaires les plus expressifs de cette nouvelle tradition anti-réhabilitation est celui de Jessica Mitford (1973). Ses attaques contre les abus de pouvoir en milieu carcéral, contre les techniques de modification du comportement, contre des institutions de type « Patuxent *, au Maryland, sont vitrioliques. La conclusion inévitable de ce genre de littérature est la prise de position catégorique pour la destruction et la disparition des institutions totalitaires, telles les prisons.

Sur un terrain moins radical, un certain nombre de criminologues s'inscrivent dans la nouvelle tradition; favorables à une utilisation très restrictive des prisons, tout en acceptant l'inévitabilité de celles-ci, ils s'élevèrent contre la thérapie obligatoire. Kittrie (1971), par exemple, parle du *droit d'être différent \. D'autres criminologues et juristes, adeptes des droits civils et des droits de l'homme, s'opposèrent à la trop grande discrétion des experts du traitement et de la réhabilitation, au nom d'une nouvelle jurisprudence des (droits des détenus , et du droit pour eux, finalement, de ne pas jouer le jeu du traitement.

Dans cette perspective, il est intéressant de mentionner le livre de Norval Morris (1974) qui, sur la base de discussions avec des praticiens chevronnés de l'Etat de l'Illinois, propose de rendre optionnel ou facultatif le traitement en institution, de définir un contrat précis et écrit avec le détenu (à son entrée en prison) où la date de sa libération conditionnelle est déjà fixée, indépendamment de son implication ou non en traitement. 
Quoi qu'il en soit, ces réactions "modérées 》 à la théorie de la réhabilitation sont elles-mêmes sous l'effet d'une contestation. En effet, le coup de grâce à la théorie fut administré par les spécialistes eux-mêmes. Les programmes de traitement en milieu institutionnel et en milieu ouvert étant fort nombreux, dans certains travaux scientifiques, relativement rigoureux, on a évalué la portée et l'efficacité de tels programmes. Or, les résultats sont très décevants à ce sujet, puisqu'ils indiquent, règle générale, qu'il n'y a pas de rapport entre le traitement et la récidive. En fait, les taux de récidive sont aussi élevés aujourd'hui qu'ils l'étaient il y a vingt-cinq, dix ou cinq ans. L'enquête la plus complète et la plus récente sur le sujet est dévastatrice pour les promoteurs des traitements de tout acabit. Cette étude de Lipton, Martinson et Wilks (1975) concluait en affirmant qu'il n'y a aucun pattern qui indique l'efficacité de quelque méthode de traitement que ce soit $\gg$. Les auteurs ont systématiquement analysé les résultats des recherches évaluatives sur le traitement en tenant compte d'une kyrielle de variables indépendantes (non seulement l'emprisonnement, mais également la probation, les libérations conditionnelles, les centres communautaires...) et dépendantes (non seulement le critère de la récidive, mais également ceux de l'ajustement institutionnel et communautaire...). Il s'agit d'une véritable encyclopédie sur l'efficacité du traitement correctionnel. Les conclusions en sont donc d'autant plus évidentes et plus accablantes pour les praticiens de la réhabilitation. A la question : "Qu'est-ce qui marche? " (What works?), la réponse des chercheurs est un magistral : « Rien ou presque.

\section{La solution? La réhabilitation de la punition (Hagg, 1975)?}

Si \& quelque chose marche ", c'est peut-être la peine plutôt que le traitement.

Cette * réhabilitation de la punition * à la mode des années 70 est d'ailleurs sanctionnée par des hommes de science de calibre. Par exemple, il y a le rapport d'un comité prestigieux * pour l'étude de l'incarcération * (Hirsch, 1975). II propose non seulement de rendre le traitement optionnel, mais d'abolir la libération conditionnelle (Hirsch, 1979). Il n'y aurait plus de sentences * ouvertes, (entre $x$ et $y$ années) ou indéterminées. Seul le principe d'équité présiderait à un régime de 
sanctions fixes et standardisées. Fogel (1975) a transcrit cette philosophie en action puisqu'il proposait un \& modèle de justice * pour l'État de l'Illinois qui s'appuie sur de telles idées. Le gouverneur de l'Illinois intervint publiquement pour appuyer le plan de Fogel. Au Canada, un des rapports de la Commission de réforme du droit du Canada (1975) s'inscrit à sa façon dans $\infty$ courant de pensée.

Ce modèle de justice est le modèle de l'heure aux ÉtatsUnis. Dejà, les codes criminels de la Californie, du Maine, de l'Indiana et de l'Illinois ont été substantiellement modifiés selon cette perspective.

La question à se poser ici est la suivante : si le modèle de justice favorise les sentences de prison fixes, diminue la discrétion des juges et abolit même dans certains cas les libérations conditionnelles, ne risque-t-il pas de faire tache d'huile et d'amener l'abolition également ou, à tout le moins, la transformation radicale de la probation?

La lecture de Corrections Magazine ainsi que l'évolution de la situation récente en Amérique du Nord dans le domaine correctionnel adulte ne justifie pas une conclusion aussi pessimiste (pessimiste pour qui ?) en ce qui concerne la probation. Du moins, quant à sa disparition. Mais quant à sa transformation, oui, on en parle.

Les agents de probation : des policiers ou des travailleurs sociaux [ déguisés 3

Si le modèle de justice est à la mode, et le modèle médical et clinique en déclin, le problème de la prison et de ses alternatives n'est pas réglé pour autant. Les études récentes sur le milieu carcéral indiquent toujours que les prisons produisent « plus de mal que de bien s. Les prisons ne réhabilitent s pas. Elles neutralisent les criminels dangereux, mais elles ne resocialisent s pas. Que faire alors de la majorité des criminels qui ne sont pas particulièrement dangereux et qui n'ont pas besoin de façon particulière de * traitement $>$ ?

La réponse automatique du criminologue à cette question est la suivante : la probation évidemment !

L'histoire pénologique (Foucault, 1975) devrait pourtant nous rendre prudents. Comme le souligne Anttila (1973), nous 
ne devons pas répéter les mêmes erreurs d'évaluation que celles commises au sujet de la valeur du traitement en milieu carcéral. $\mathrm{Si}$, de nouveau, nous essayons de trouver un remède-panacée pour tous les cas, nous nous acheminons vers un autre échec. Si nous croyons encore à un système de sanction, telle la probation, capable d'atteindre simultanément des objectifs aussi difficiles que l'effet intimidant de la peine (en général et en particulier) ainsi que la réhabilitation d'un individu, nous ne ferons rien de plus que de transférer nos attentes et nos espérances inflationnaires d'une structure (prison) à l'autre (probation). Une politique de contrôle de la criminalité n'est malheureusement pas aussi simple.

Même la terminologie utilisée est trompeuse. La probation, dit-on, c'est une mesure de « traitement en milieu ouvert ». Le

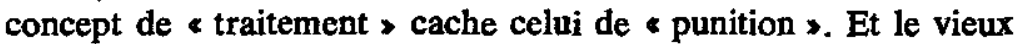
refrain revient : « donnez-nous plus d'argent, plus de ressources, plus d'équipement...

Ce raisonnement est dépassé. Il n'y aura pas de miracle. Aucun système spécifique de politique criminelle ne changera drastiquement les situations criminogènes. Le système pénal n'affecte qu'en partie le niveau et le développement de la criminalité. Le niveau et de développement socio-économique et culturel d'une société sont des variables plus importantes.

Comme le disait Durkheim, toute société a néanmoins besoin de sanctions. Mais quelles sanctions? D'accord, les sanctions en milieu ouvert sont meilleures, non pas nécessairement sur le plan de l'efficacité mais à tout le moins sur un simple plan humanitaire. Soit. A remarquer que sur ce plan, les amendes, la restitution, les travaux communautaires... sont encore de bien meilleures formules (quoique l'on peut assumer sous la probation la restitution et les travaux communautaires).

Une sanction « normale », donc, devrait répondre aux indicateurs suivants :

a) elle ne doit pas être de nature carcérale;

b) elle ne doit pas apparaître comme étant trop sévère, ou trop indulgente ;

c) elle ne doit pas être trop coûteuse pour la société et les ressources matérielles disponibles doivent être transférées aux systèmes de services sociaux; 
d) elle ne doit pas spécialement faire appel à un personnel trop spécialisé ;

e) elle doit être appliquée de la façon la plus simple possible et aussitôt la décision rendue par le tribunal.

La probation correspond à ces critères si, d'abord et avant tout, elle est définie en termes d'obligation pour le probationnaire de se rapporter à l'agent de probation. Cette obligation est un contrôle, au sens propre du terme; elle est donc de nature disciplinaire et punitive. Il est correct qu'il en soit ainsi. Le traitement ou le counselling clinique ne doivent pas être une fonction de l'agent de probation. Ces services doivent être rendus par les agences sociales ordinaires au sein de la société. Ces services sociaux, de plus, doivent être offerts sur une base volontaire sans trace de coercition.

Le mouvement des idées sur la probation au cours des dernières années nous incite ainsi à bien distinguer la punition et le traitement. Utilisons à la fois * la punition en milieu ouvert * et cle service social en milieu ouvert \. Mais les deux orientations ne doivent pas être fusionnées en une seule, ce qui est souvent le cas aujourd'hui.

La littérature nord-américaine récente nous livre un message clair et précis : l'agent de probation des années 80 sera un agent de contrôle et non pas un agent de contrôle et de traitement, simultanément.

Toutefois, ce rôle est-il suffisant ? N'est-il pas trop dévalorisant pour l'agent de probation? Nous ne le croyons pas si le rôle de contrôle de l'agent est complété par un rôle de * négociateur de ressources s.

\section{Les agents de probation : des négociateurs de ressources?}

Si l'agent de probation doit choisir ainsi entre son rôle de police et son rôle de travailleur social, il se pourrait bien qu'il décide de ne choisir ni l'un ni l'autre. Il pourrait trouver une - troisième voie , qui serait celle d'un négociateur de ressources, responsable de pairer les besoins de ses clients avec les programmes communautaires aptes à $\mathrm{y}$ répondre et à $\mathrm{y}$ satisfaire.

Ce nouveau concept de e négociateur > (Wilson, 1978) fait son petit bonhomme de chemin aux États-Unis depuis près de 
deux ans. L'on parle ainsi, à la mode américaine, du C.R.M.T. (Community Resources Management Team). Les agents travaillent en équipe, avec des grappes de cas (caseloads). L'a.b.c. de la probation est ainsi respecté : l'agent est un \& plaideur > pour son client, un «négociateur de ressources > et une personnecontact ou agent de liaison avec la e communauté s.

Cette métamorphose du rôle de l'agent de probation est importante. Ni policier, ni travailleur social, mais un * négociateur de ressources, qui essaie de pairer les besoins de ses clients avec les programmes communautaires et les ressources des services sociaux. L'agent diminue le temps-travail au niveau du counselling et de la surveillance mais il en accorde beaucoup plus pour obtenir la collaboration du personnel des agences sociales et pour persuader son client de jouer le jeu et d'accepter de travailler avec ces agences.

L'agent de probation n'aura plus ses propres cas à lui tout seul mais les partagera avec d'autres agents. Ensemble, en équipe de cinq à dix agents, des décisions seront prises. L'agent n'essaiera plus d'être le e spécialiste > pour tous ses clients, mais il se spécialisera dans un domaine particulier (toxicomanie, santé mentale, main-d'œuvre...).

Le concept de e négociateur s a été développé il y a quelques années par un groupe du Colorado au nom d'un consortium de treize (13) états du Sud-Ouest des Etats-Unis.

L'évaluation préliminaire (qui doit être suivie d'une évaluation rigoureuse en profondeur) démontre l'efficacité de cette nouvelle approche. Les clients sont * mieux servis > et * mieux suivis ». Mais il n'y a pas de preuve encore au sujet de la diminution de la récidive.

Cette remise en question du rôle traditionnel de l'agent de probation est évidemment * menaçante ». Aussi, certaines agences de probation n'ont pas * acheté , en bloc les quatre éléments principaux de cette réforme mais en ont sélectionné un ou deux selon leurs besoins, à savoir : l'évaluation des besoins, la négociation des ressources, les grappes de cas et la gestion en équipe. Il semble, toutefois, que l'approche partielle n'est pas aussi satisfaisante que l'approche globale.

Une équipe typique est constituée d'un responsable, de cinq agents de probation et d'une grappe de 400 clients. Environ 
$40 \%$ de ces cas sont des cas symboliques seulement. Des visites occasionnelles à l'agence sont suffisantes et l'utilisation de bénévoles-para-professionnels est une formule adéquate pour ces clients.

Les autres sont de * vrais > cas (toxicomanie, emploi, santé mentale et physique, lacunes éducationnelles...). Chaque agent se spécialise dans un secteur ou l'autre. Mais e se spécialiser , ne signifie pas e être un expert >; ça signifie plutôt que chaque agent connaît bien les programmes et les agences communautaires spécialisées dans son secteur, comme l'agent d'immeuble connaît ce qui est disponible sur le marché immobilier. Le client peut évidemment avoir besoin de plusieurs types de services et de ressources. Le travail en équipe permet de s'en occuper convenablement, même si un agent se charge de façon particulière de son besoin le plus important et le plus immédiat.

Chaque agent doit ainsi développer une filière des ressources communautaires et il doit passer une partie importante de son temps \& sur le terrain > à visiter et à contacter les programmes et les personnes-ressources ainsi qu'à découvrir le $<$ bon programme " pour répondre aux besoins du client. L' \& expert * est donc sur le terrain et l'agent de probation lui confie un client en l'assurant de son appui, en essayant de convaincre et de vaincre la résistance du client à son égard. Par la même occasion, toutefois, l'agent rend responsable l'expert de la prise en charge de son client.

Les contacts personnels entre les agents de probation et les agences sont excessivement importants. Ils doivent être réguliers et suivis. Faits de bonne foi et en confiance mutuelle. C'est à l'avantage de tout le monde. Mais ce n'est pas facile. Un effort soutenu doit être consenti.

Le rôle de * plaideur * pour un client est un des plus difficiles à accomplir. L'agent doit alors dépasser les résistances non verbales, subtiles et défier les attitudes négatives du public envers le criminel. La résistance vient également de certains agents de probation qui sont dérangés, par cette nouvelle perspective.

Du côté communautaire, les spécialistes qui ont participé jusqu'ici à ce programme en sont généralement fort heureux. 
L'autorité officielle conférée à l'agent de probation lui permet de persuader un client récalcitrant à coopérer avec lui.

Soulignons que les agents de probation ont en fait plus de contacts avec le personnel et les spécialistes des agences qu'avec leurs clients. Souvent, des rencontres de groupes ont lieu entre les agents et l'ensemble des responsables des agences sociales. L'on développe ainsi une «coalition * d'intérêts apte à mieux servir les clients et à éviter cette " guerre de tranchées > où chacun reste dans son coin à cause d'une jalousie morbide et de l'instinct territorial de la protection de son fief.

Evidemment, une telle approche implique un changement important dans la relation entre l'agent de probation et son client. Il s'agit d'une relation d'affaire, plus systématique, de nature procédurière. Certains agents ont alors l'impression de ne plus avoir de relation interpersonnelle traditionnelle avec le client. Leur fierté professionnelle en est affectée. Abandonner l'image d'un agent habile à changer lui-même les attitudes et le comportement du client n'est pas très apprécié, cela va sans dire. Admettre que cette relation interpersonnelle n'est peut-être pas productive et que cette relation est peut-être plus importante pour eux que pour le client n'est pas facile.

Ce nouveau rôle de négociateur de ressources qui assure la liaison entre les clients et les experts sur le terrain est donc un projet qui n'ira pas sans peine.

Mais la remise en question actuellement du secteur correctionnel adulte indique qu'en ce qui concerne la probation, ce rôle sera celui des années 80 .

\section{Conclusion}

Il n'est pas besoin d'être un criminologue critique radical ou novateur (Taylor et al, 1973) pour s'apercevoir que le secteur correctionnel adulte en Europe comme en Amérique du Nord est contesté et que la perspective abolitionniste a ouvert des brèches dans ce domaine de nos activités professionnelles traditionnelles. Mais il ne faudrait pas «partir en peur > et créer l'impression que ce qui a été fait jusqu'ici par les agents de probation et les autres acteurs du système correctionnel est à rejeter du revers de la main. Après tout, il ne s'agit pas d'une « bataille à finir > entre la gauche et la droite, entre les radicaux 
et les conservateurs. En effet, même la gauche criminologique et les criminologues radicaux acceptent l'inévitabilité d'un certain contrôle social, mais leur clientèle-cible est souvent différente (criminalité économique des \& puissants , versus criminalité classique des classes sociales défavorisées).

Dans cette perspective, l'interrogation en théorie et en pratique du rôle d'agent de contrôle social en probation, par exemple, est saine. Mais la vérité à ce sujet ne sortira pas de la bouche des enfants. Elle émanera de la praxis des agents au cours des prochaines années ainsi que l'évolution plus globale du système juridique et du système socio-politique et socio-économique de nos sociétés occidentales.

A nous de participer aux débats et de renouveler la pratique criminologique en conséquence. 


\section{BIBLIOGRAPHIE}

CORRECTIONS MAGAZINE (depuis 1974). Le lecteur peut s'abonner à cette revue en écrivant au 801 Second Avenue, New York, N.Y. 10017. L'abonnement annuel est de $\$ 18$ U.S.

ANTTILA, I. (1975) : Probation and parole: social control or social service *, International Journal of Criminology and Penology, 3, $79,84$.

COMMMISSION DE REFORME DU DROTT DU CANADA (1975) : L'emprisonnement et la libération, Ottawa, Information-Canada.

FOGEL, D. (1975) : We are the living proof : the justice model for corrections, Cincinnati, W.H. Anderson Co.

FOUCAULT, M. (1975) : Surveller et punir : naissance de la prison, Paris, Gallimard.

HAAG, E. Van Den (1975) : Punishing criminals, New York, Basic Books.

HIRSCH, A. (1975) : Doing justice : the choice of punishments, New York, Hill and Wang.

HIRSCH, A. (1979) : Abolish parole, Massachusetts, Ballinger.

KITTRIE, N.. (1971) : The right to be different : deviance and enforced therapy, Baltimore, Johns Hopkins Press.

LIPTON, D., R. MARTINSON and J. WILKS (1975) : The effectiveness of correctional treatment : a survey of treatment evaluation studies, New York, Praeger.

MrTFORD, J. (1973) : Kind and usual punishment, New York, Knopf.

MORRIS, N. (1974) : The future of imprisonment, Chicago, University of Chicago Press.

TAYLOR, I., P. WALTON and J. YOUNG (1973) : The new-criminology, London, Routledge and Kegan Paul.

WILSON, R. (1978) : C Probation/Parole officers as a resource brokers s, Corrections Magazine, 2, 48-54. 